\title{
P 133 HOW COMMON IS DELIRIUM IN PALLIATIVE CARE INPATIENT UNITS AND WHAT IS THE OUTCOME FOR THESE PATIENTS?
}

Anna Porteous, Felicity Dewhurst, Eleanor Grogan, Lucy Lowery, Fiona MacCormick, Ann Paxton, Jennifer Vidrine, Rowan Walmsley. Health Education North East, Newcastle Upon Tyne, UK

10.1136/bmjspcare-2014-000654.174

Background Delirium affects up to $50 \%$ of patients on medical wards (NICE), however the impact in palliative care units is unclear, with prevalence estimates ranging from 13-64\%. Use of delirium screening tools in palliative care is inconsistent and consensus opinion is lacking. The short-CAM is a wellresearched, observational tool, recommended by NICE in high-risk in-patients. It is often favoured for its ease of use and inter-rater reliability and is one of a limited number to be validated in the palliative care setting.

Aim To identify the prevalence, incidence and outcome of delirium in five specialist palliative care inpatient units (SPCUs) in the North East using the short-CAM.

Methods Adult patients admitted to five SPCUs were screened for delirium using the short-CAM. Patients with significant communication barriers were excluded. Screening occurred on admission, weekly and if clinical change raised the suspicion of delirium. Anonymised patient data was collected by clinical staff based on the units between April and August 2013.

Results Two hundred and ninety-one patients were screened for delirium using the short-CAM during the study period. Thirty-four (11.7\%) patients were delirious (CAM-positive) on admission to the SPCUs. Twenty-four patients $(8.2 \%)$ became delirious during their admission. Resolution of delirium occurred in $38.6 \%$ of cases. Of 126 patients who died during their admission, 58 (46\%) had been delirious prior to death.

Conclusion Overall, delirium (as identified by the short-CAM) affected $20 \%$ of patients included in this study. Our findings demonstrate a lower prevalence and incidence of delirium amongst palliative care patients than many published studies. This may relate to diagnostic limitations of the short-CAM, as well as variations in the sensitivity of screening tools employed in these studies. It is clear that delirium is associated with poor prognosis. Further studies to explore the impact and management of this distressing condition are warranted. 